\title{
Evaluating the Impact of Fracture-Induced Anisotropy on Reservoir Rock Property Estimates made from Seismic Data
}

\author{
Boris Gurevich and Benn Hansen \\ Dept. of Exploration Geophysics, Curtin University of Technology, Perth, Australia
}

\begin{abstract}
Naturally fractured reservoirs have attracted increasing interest in exploration and production geophysics in recent years. The presence of fractures can affect the AVO response of a reservoir. In this paper we analyse this effect of fractures in a porous reservoir on seismic data, and estimate the errors in the estimation of reservoir properties due to ignoring the presence of these fractures.

The effect of fractures on elastic properties of a porous rock is studied using the model of fractures as linear-slip interfaces in an isotropic porous background. The effect of fluid on the elastic properties of this medium is modelled using equations of anisotropic poroelasticity. This yields explicit analytical expressions for the elastic properties of the fractured porous medium saturated with a given fluid.

We use this theory to model the seismic response of a reservoir with vertical fractures. We then invert this response for reservoir properties (porosity, net-to-gross) using a workflow based on the above theory, and compare the predictions with the results of the standard industry workflow based on the isotropic Gassmann equation. The comparison yields an estimate of the potential error in the prediction of rock properties using an isotropic workflow for a fractured reservoir. These errors can be as large as $\pm 3 \%$ in predicted porosity, and $\pm 40 \%$ in predicted netto-gross.
\end{abstract}

\section{Introduction}

The main application of the surface seismic method in the appraisal and production environment is in reservoir characterisation. To target high porosity and net-to-gross reservoir intervals, an assessment of reservoir rock properties is required prior to drilling. To achieve this purpose, we need an accurate estimation of subsurface rock properties from surface seismic data.

The common method of determining subsurface lithology from surface seismic involves an amplitude versus offset (AVO) inversion process, to convert seismic amplitude and offset information into high frequency impedance data. This data is then merged with low frequency impedance trends derived from well logs, and the inverted impedance traces are calibrated at the well location. Well log derived rock property trends are then applied to this impedance data to make reservoir property predictions. These trends are relations between acoustic impedance, shear impedance and rock properties, such as porosity, net-to-gross ratio, and fluid fill. They provide the link between inverted seismic impedance data and subsurface geology.

The standard workflow for producing these rock property trends assumes an isotropic reservoir and thus ignores the effects of anisotropy. Rock property predictions made using this isotropic workflow will be incorrect if the reservoir is anisotropic. Incorrect rock property predictions will have a significant effect on reservoir volumetric calculations, and will therefore impact field development decisions.

Reservoir anisotropy may be caused by the alignment of clay particles in shales, intrareservoir layering or reservoir fracturing. In this study, the effect of fractures on the estimation of rock properties in a porous laminated sand-shale reservoir was analysed. Both isotropic and anisotropic rock property prediction workflows were constructed and used to estimate rock properties from one input seismic impedance dataset. From these predictions, the potential error 
in the rock properties estimated using an isotropic workflow for a fractured sand-shale reservoir was quantified.

\section{Isotropic Rock Property Prediction Workflow}

The initial step in producing the rock property trends involves well log data from within the reservoir interval. A set of P-wave velocity $\left(V_{P}\right)$, S-wave velocity $\left(V_{S}\right)$, and density $(\rho)$ measurements for a range of reservoir porosities is obtained from within a clean (high net-togross), brine-saturated sand interval. This data is used to produce three brine saturated sand trends: $V_{P}$ versus porosity, $V_{S}$ versus porosity, and density versus porosity. As we are using a laminated sand-shale reservoir model, representative "mixing shale" properties are also required from the well logs. It is generally accepted that shale fluid saturation can not be changed within a production timescale, and therefore the shale component is assumed to have zero effective porosity, and an intrinsic brine saturation. The mixing shale's elastic properties are therefore invariant with both porosity and fluid fill, and can be represented completely by a single set of $V_{P}, V_{S}$ and $\rho$ values. These are derived from well $\log$ data within a high shale content (low netto-gross) interval.

Changes in reservoir fluid fill can be represented by a change in the fluid saturation of the sandstone only. Therefore fluid substitution is applied to the brine saturated sandstone properties prior to mixing of the sand and shale components.

From the brine saturated sandstone velocity and density trends the saturated bulk and shear moduli ( $K_{\text {sat }}$ and $\mu_{\text {sat }}$ respectively) are determined. Using these saturated elastic moduli, together with a brine fluid modulus $K_{f}$ and a sandstone grain modulus $K_{g}$ (measured from fluid and core samples respectively), the dry rock bulk modulus as a function of porosity $\phi$ can be obtained by solving the Gassmann equation (White, 1965):

$$
K_{s a t}=K_{g} \frac{\phi K_{d r y}-\frac{(1+\phi) K_{f} K_{d r y}}{K_{g}}+K_{f}}{(1+\phi) K_{f}+\phi K_{g}-\frac{K_{f} K_{d r y}}{K_{g}}} .
$$

Equation (1) can then be used to perform fluid substitution to produce trends of hydrocarbon saturated bulk moduli versus porosity. The shear modulus of the saturated rock is independent of the saturating fluid, and the density is given by a porosity-weighted average.

The Gassmann equation is based on the assumption that the saturated rock is composed of only one mineral phase, and that there is only one saturating fluid. The assumption of one mineral phase is adequate for pure sandstone, but a multi-fluid partial saturation model is required. This was provided by the Reuss isostress mixing law, which enables the bulk modulus of a fluid composed of multiple fluid phases (in this case brine and hydrocarbon) to be calculated as the weighted harmonic average of the component fluid bulk moduli, Domenico (1976):

$$
\frac{1}{K_{f(e f f)}}=\frac{S_{w}}{K_{w}}+\frac{1-S_{w}}{K_{h c}}
$$

where $K_{f(\text { eff })}$ is the effective fluid bulk modulus, $S_{w}$ the brine saturation, $K_{w}$ the brine bulk modulus and $K_{h c}$ the hydrocarbon bulk modulus. The mixed phase fluid density is given by a volumetric weighted average of the fluid component densities.

After fluid substitution of the sandstone component, the elastic moduli of the thinly layered sand/shale sequence can be obtained by Backus averaging of the shale and saturated sand 
moduli (Mavko et al., 1998). From these moduli, the laminated reservoir equivalent $V_{P}, V_{S}$ and $\rho$ values can be determined, to derive trends of acoustic impedance (AI), shear impedance (SI) and $V_{P} / V_{S}$ versus porosity and net-to-gross for various reservoir fluid saturations. These trends can then be used to estimate reservoir rock properties from seismic data.

Whilst this workflow is effective in predicting rock properties for an isotropic reservoir, it is not strictly valid in cases where anisotropy is present, such as in a fractured reservoir. In such a case, the workflow may produce incorrect estimated porosity, net-to-gross and fluid fill values, as the isotropic Gassmann equations and Backus sand-shale mixing do not apply. In anisotropic reservoir situations, an anisotropic rock property prediction workflow is required.

\section{Anisotropic Rock Property Prediction Workflow}

The anisotropic workflow begins in a similar fashion to the isotropic workflow, with well $\log$ data from within the reservoir interval. This well log data is used to derive "mixing shale" $V_{P}, V_{S}$ and density values (which are invariant with porosity), and velocity and density trends versus porosity for the brine saturated sandstone.

Additional information required from the well $\operatorname{logs}$ in the anisotropic workflow is a measurement of the degree of anisotropy present within the fractured sandstone. This can be expressed in the form of Thomsen's (1986) shear-wave splitting parameter $\gamma$. In a well logging situation this parameter can be evaluated through the use of a cross-dipole sonic tool. This tool contains two perpendicularly polarised dipole sources, and two perpendicularly polarised dipole receivers. In vertically fractured (HTI) media, a vertically incident S-wave splits into two perpendicularly polarised S-wave components, a slow S-wave polarised perpendicular to the fracture planes, and a fast S-wave polarised parallel to the fracture planes. The dipole sonic tool introduces a vertically incident S-wave into the borehole, and its perpendicular receivers capture varying components of each of the polarised S-waves, dependent on the tool's orientation. Processing is then required to mathematically rotate the tool records so that the two S-wave components are split one onto each receiver. Thomsen's $\gamma$ parameter can then be estimated from the two S-wave velocities, using the equation:

$$
\gamma=\frac{V_{\text {fast }}-V_{\text {slow }}}{V_{\text {slow }}} .
$$

The fracture orientation can also be determined, as this is the orientation of the rotated receiver which registers the fast S-wave arrival.

In a manner identical to the isotropic workflow, the brine-saturated sandstone velocity and density trends are used to calculate the brine saturated shear and bulk moduli. However, the isotropic Gassmann equations can not be used to solve for the dry rock properties. Instead an anisotropic approach must be taken, using the anisotropic Gassmann (1951) fluid substitution discussed by Gurevich (2002).

Gurevich (2002) uses a transversely isotropic medium with a horizontal axis of symmetry (HTI) to represent an isotropic background rock hosting a series of vertical, parallel, rotationally invariant fractures. The dry fractured porous medium can then be represented by the general linear-slip model of a medium with parallel fractures (Schoenberg and Douma, 1988; Schoenberg and Sayers, 1995). This model uses physically intuitive relations between stress and discontinuity in displacement across the fracture plane, and is formulated in terms of excess compliances (or weaknesses) of the material caused by the presence of fractures. It requires no assumptions about the microstructure or microgeometry of the fractures (Bakulin et al., 2000). 
Within the assumptions of the linear-slip model the fractured medium with rotationally invariant vertical fractures is transversely isotropic with a horizontal symmetry axis. A general transversely isotropic medium is described by five independent elastic parameters. However, in the case of an isotropic medium with rotationally invariant parallel fractures, only four parameters are required: the two elastic constants of the isotropic host medium $\lambda$ and $\mu$, and the two fracture weaknesses $\Delta_{N}$ and $\Delta_{T}$ (or the two fracture excess compliances $Z_{N}$ and $Z_{T}$ ). From these parameters of the dry fractured rock, we can derive the anisotropic elastic moduli of the saturated rock by using the anisotropic Gassmann equations (Gurevich, 2002):

$$
\begin{aligned}
& c_{11}^{\text {sat }}=\frac{L}{D}\left\{d_{1} \theta+\frac{K_{f}}{\phi K_{g} L}\left[L_{1} \eta^{\prime}-\frac{16}{9} \frac{\mu^{2} \eta_{0}}{L} \Delta_{N}\right]\right\}, \\
& c_{33}^{\text {sat }}=\frac{L}{D}\left\{d_{2} \theta+\frac{K_{f}}{\phi K_{g} L}\left[L_{1} \eta^{\prime}-\frac{4}{9} \frac{\mu^{2} \eta_{0}}{L} \Delta_{N}\right]\right\}, \\
& c_{13}^{\text {sat }}=\frac{\lambda}{D}\left\{d_{1} \theta+\frac{K_{f}}{\phi K_{g} \lambda}\left[\lambda_{1} \eta^{\prime}+\frac{8}{9} \frac{\mu^{2} \eta_{0}}{L} \Delta_{N}\right]\right\}, \\
& c_{23}^{\text {sat }}=\frac{\lambda}{D}\left\{\left(1-\frac{\lambda}{L} \Delta_{N}\right) \theta+\frac{K_{f}}{\phi K_{g} \lambda}\left[\lambda_{1} \eta^{\prime}-\frac{4}{9} \frac{\mu^{2} \eta_{0}}{L} \Delta_{N}\right]\right\}, \\
& c_{44}^{\text {sat }}=\mu \quad \text { and } \quad c_{55}^{\text {sat }}=\mu\left(1-\Delta_{T}\right),
\end{aligned}
$$

where the following notation was used

$$
\begin{array}{ll}
D=1+\frac{K_{f}}{K_{g} \phi}\left(\eta_{0}-\phi+\frac{K^{2} \Delta_{N}}{K_{g} L}\right), & \theta=1-\frac{K_{f}}{K_{g}}, \quad \eta^{\prime}=\eta_{0}+\frac{K^{2}}{K_{g} L} \Delta_{N}, \\
L_{1}=K_{g}+\frac{4}{3} \mu, \quad \lambda_{1}=K_{g}-\frac{2}{3} \mu, & d_{1}=1-\Delta_{N}, \quad d_{2}=1-\frac{\lambda^{2}}{L^{2}} \Delta_{N}, \\
L=\lambda+2 \mu, & \eta_{0}=1-\frac{K}{K_{g}}, \quad \text { and } \quad K=\lambda+\frac{2 \mu}{3},
\end{array}
$$

$K$ is the dry host bulk modulus and $\lambda$ a Lamé parameter of the dry isotropic host.

These equations provide a complete description of the elastic properties of the saturated porous rock with aligned fractures and serve as an anisotropic equivalent to conventional isotropic Gassmann fluid substitution.

In terms of application within our anisotropic rock property prediction workflow, Thomsen's shear wave splitting parameter $\gamma$ is known from the dipole sonic log, and can be used to calculate the dry rock tangential fracture compliance (Schoenberg and Douma, 1988):

$$
Z_{T}=\frac{2 \gamma}{\mu} \text {. }
$$

Due to the limitations of the data available, it is possible to evaluate only $Z_{T}$. Here we assume the normal fracture compliance $Z_{N}$ to be equal to $Z_{T}$. This assumption is thought to be reasonable for dry rocks (Schoenberg and Sayers, 1995).

The next step is to determine the dry background bulk modulus $K$. This is done by first evaluating the saturated rock stiffness matrix component $c_{33}^{\text {sat }}$ from the well log density and 
vertical P-wave velocity $V_{p(z)}$,

$$
c_{33}^{\text {sat }}=V_{P(Z)}^{2} \rho .
$$

The dry background bulk modulus can then be obtained by solving equation (5) and the normal fracture weakness $\Delta_{N}$ can be calculated from the equation

$$
\Delta_{N}=\frac{\left(K+\frac{4}{3} \mu\right) Z_{N}}{1+\left(K+\frac{4}{3} \mu\right) Z_{N}} .
$$

We can now obtain the saturated rock stiffness matrices from the moduli of the dry rock and fracture parameters. To produce the hydrocarbon saturated sandstone elastic moduli we substitute the known fracture parameters into equations (4) - (8) with the bulk moduli of the required saturating fluids, to calculate the saturated rock stiffness matrix components $c_{11}, c_{33}, c_{13}$, $c_{23}, c_{44}$ and $c_{55}$. As the fractured rock is known to be HTI, the remaining components of the stiffness matrices can be populated in accordance with the HTI stiffness matrix structure. A porosity and fluid fill independent shale stiffness matrix is also constructed, using reservoir shale $V_{P}, V_{S}$ and density values, along with known shale anisotropic parameters.

The reservoir under consideration is a system of thin VTI shale and HTI fractured sand layers. To estimate the elastic properties of such sequence, a mixing law that takes into account azimuthal anisotropy of the constitutive layers is required. Such a mixing law is given by Schoenberg and Muir (1989) and shows that the resulting mixed laminated sand-shale reservoir is orthorhombic, with two perpendicular axes of symmetry.

As a result of the mixing procedure, we obtain stiffness matrices for each porosity and fluid saturation, describing the change in elastic properties with varying reservoir net-to-gross. For comparison with the isotropic workflow results, these matrices can be used to compute vertical P-wave velocities and the velocities of vertically incident $\mathrm{S}$-waves polarised in different azimuths (Mavko et al. 1998).

However, these vertical velocities are not suitable to compare to the $V_{P}$ and $V_{S}$ values derived from AVO inverted seismic, as the industry standard AVO inversion uses an isotropic AVO approximation. The velocities obtained from AVO inversion will be the isotropic AVO equivalent' of the anisotropic reservoir velocities.

To obtain these 'isotropic AVO equivalent' velocities, an anisotropic AVO approximation is required. For P-waves in orthorhombic media, the short-offset approximation is given by Rüger (1998):

$$
R_{P}(\omega, \varphi)=\frac{1}{2} \frac{\Delta Z}{\bar{Z}}+\frac{1}{2}\left\{\frac{\Delta \alpha}{\bar{\alpha}}-\left(\frac{2 \bar{\beta}}{\bar{\alpha}}\right)^{2} \frac{\Delta \mu}{\bar{\mu}}+\left[\Delta \delta^{(2)}+2\left(\frac{2 \bar{\beta}}{\bar{\alpha}}\right)^{2} \Delta \gamma\right] \cos ^{2} \varphi+\Delta \delta^{(1)} \sin ^{2} \varphi\right\} \sin ^{2} \omega
$$

In equation (46), $\varphi$ is the azimuthal angle between the wave vector and the horizontal symmetry axis, $\alpha$ is the $\mathrm{P}$-wave velocity, $\beta$ the $\mathrm{S}$-wave velocity, $Z=\rho \alpha$ the $\mathrm{P}$-wave impedance, $\Delta x=x_{2}-x_{1}$ and $\bar{x}=\left(x_{1}+x_{2}\right) / 2$ (where $x_{1}$ is an overburden property and $x_{2}$ is a reservoir property), $\delta^{2)}$ is the VTI parameter $\delta$ observed in the $\left[x_{1}, x_{3}\right]$ plane, and $\delta^{l)}$ is the VTI parameter $\delta$, in the $\left[x_{2}, x_{3}\right]$ plane. These two parameters can be determined from the orthorhombic stiffness matrix using the equations

$$
\delta^{(1)}=\frac{\left(c_{23}+c_{44}\right)^{2}-\left(c_{33}-c_{44}\right)^{2}}{2 c_{33}\left(c_{33}-c_{44}\right)} ; \quad \delta^{(2)}=\frac{\left(c_{13}+c_{55}\right)^{2}-\left(c_{33}-c_{55}\right)^{2}}{2 c_{33}\left(c_{33}-c_{55}\right)} .
$$

Equation (15) shows that the zero-offset reflectivity is a function of only the acoustic 
impedance contrast between the overburden and the reservoir, and is unaffected by reservoir anisotropy. This is an identical situation to the isotropic AVO approximation. Therefore the vertical $V_{P}$ obtained from the reservoir orthorhombic stiffness matrix is equal to $V_{P}$ obtained from isotropic AVO inversion, which is derived solely from the zero-offset reflectivity term.

In contrast, the isotropic and anisotropic 'gradient' terms are not identical. The 'isotropic $\mathrm{AVO}$ equivalent' S-wave velocity $\beta_{\text {eff }}$ is determined by equating the isotropic and anisotropic gradient terms:

$$
\left(\frac{2 \overline{\beta_{e f f}}}{\bar{\alpha}}\right)^{2} \frac{\Delta \mu_{e f f}}{\bar{\mu}}=\left(\frac{2 \bar{\beta}}{\bar{\alpha}}\right)^{2} \frac{\Delta \mu}{\bar{\mu}}+\left[\Delta \delta^{(2)}+2\left(\frac{2 \bar{\beta}}{\bar{\alpha}}\right)^{2} \Delta \gamma\right] \cos ^{2} \varphi+\Delta \delta^{(1)} \sin ^{2} \varphi,
$$

and solving. This produces an azimuthally dependent S-wave velocity, which incorporates both $\mathrm{S}$-wave and P-wave anisotropy effects at moderate offsets.

Finally, the obtained P-wave and apparent S-wave velocities can be used to derive the rock property trends in terms of acoustic impedance and $V_{P} / V_{S}$ for brine-, oil- and gas-saturated fractured sand-shale reservoirs, and these trends can be applied to inverted impedance data.

\section{Synthetic Dataset}

To demonstrate the difference in the rock property predictions by the two workflows, we applied both of them to a dataset provided by Woodside Energy Ltd. The dataset consisted of reservoir mineral and fluid properties, brine saturated sand velocity-porosity and density-porosity trends, a dipole sonic well log, and a seismic survey azimuth. The shear wave splitting parameter $\gamma$ estimated from the dipole sonic $\log$ was $10 \%$, and the fracture azimuth was $300^{\circ}$. The seismic acquisition azimuth over the well location was $90^{\circ}$ from North, and therefore the angle $\varphi$ between the fracture normal and the seismic acquisition azimuth was $60^{\circ}$.

Acoustic impedance (AI) and $V_{P} / V_{S}$ values were required to input to both rock property prediction workflows. These values were obtained by using the anisotropic workflow to produce rock property trends in terms of $\mathrm{AI}$ and $V_{P} / V_{S}$ from the input well log velocity and density versus porosity trends. These trends therefore simulated seismically realistic $\mathrm{AI}$ and $V_{P} / V_{S}$ results for a fractured reservoir. The advantage of this approach was that the correct porosity, net-to-gross and fluid fill values for each of the input $\mathrm{AI}$ and $V_{P} / V_{S}$ values was known. This meant that the anisotropic workflow yielded correct rock property estimates, whilst the isotropic workflow demonstrated the situations where the industry workflow would be in error, and provided an estimate of the size of this mismatch.

\section{Results}

Figures 1.4 to 1.9 show the absolute error in the isotropic predicted porosity and net-togross values for the three fluid saturations in terms of expected porosity and expected net-togross. The absolute error was the absolute value of the difference between the anisotropic and isotropic predicted rock properties.

The maximum porosity prediction error was 3\% and occurred in the oil saturated case. The absolute error in the isotropic predicted porosities for the brine and gas saturated reservoirs were $2.5 \%$ and $2 \%$ respectively. However, the error made in net-to-gross prediction was much larger. The maximum error was almost $40 \%$ in the brine saturated case and the oil and gas saturated maximum errors were $30 \%$ and $25 \%$ respectively. 


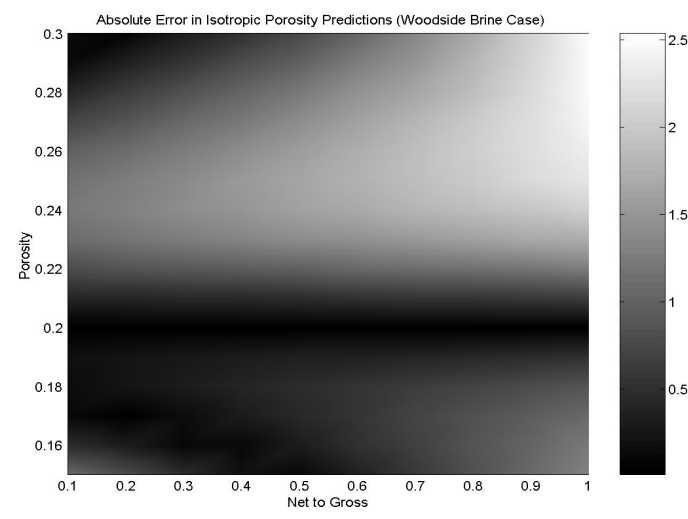

Figure 1.4 - Absolute error in isotropic porosity predictions for Woodside brine saturated reservoir

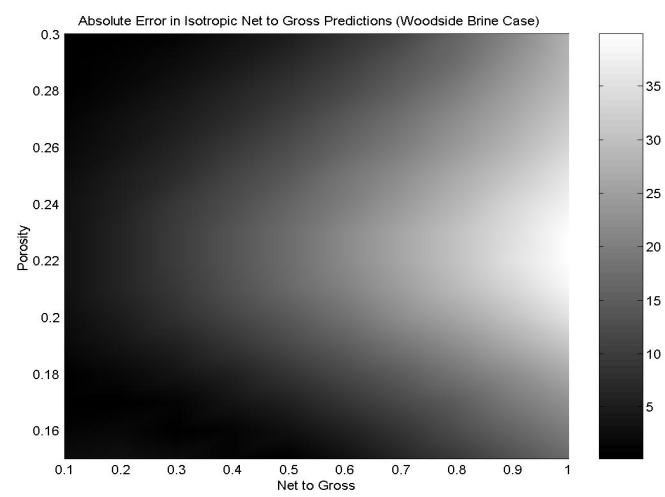

Figure 1.5 - Absolute error in isotropic net-to-gross predictions for Woodside brine saturated reservoir

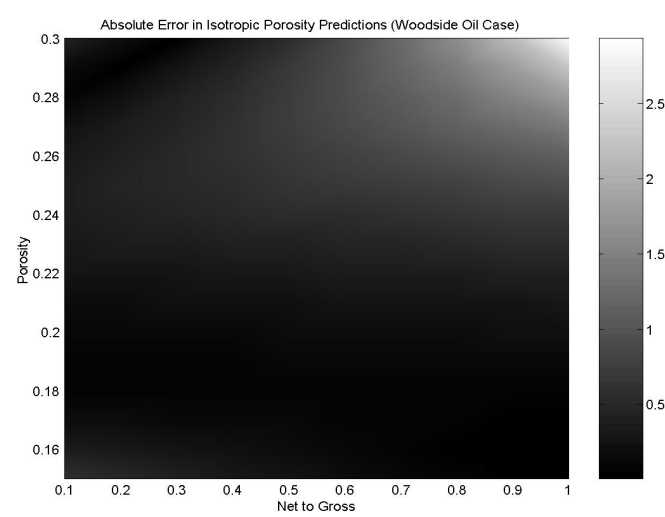

Figure 1.6 - Absolute error in isotropic porosity predictions for Woodside oil saturated reservoir

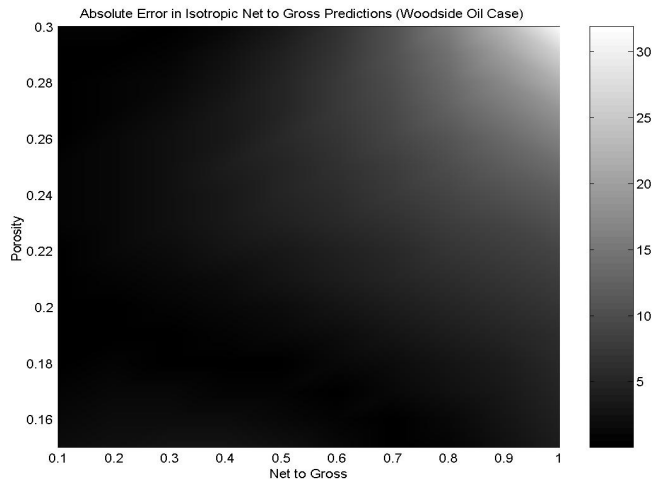

Figure 1.7 - Absolute error in isotropic net-to-gross predictions for Woodside oil saturated reservoir

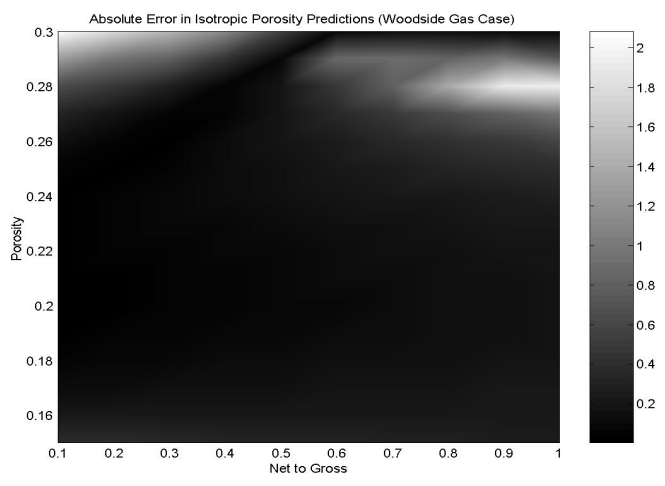

Figure 1.8 - Absolute error in isotropic porosity predictions for Woodside gas saturated reservoir

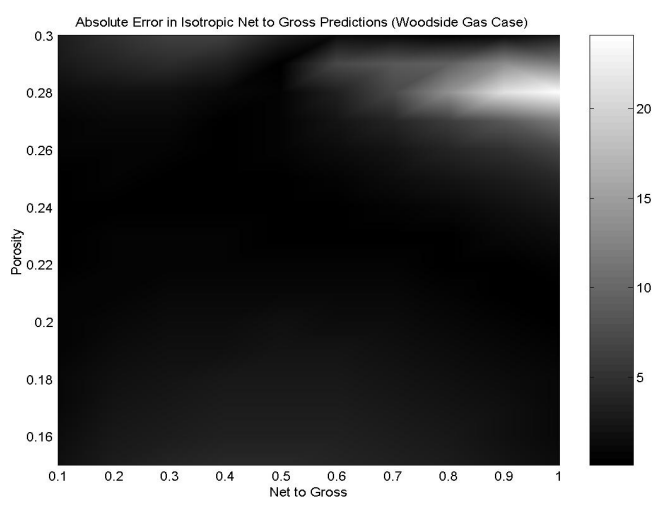

Figure 1.9 - Absolute error in isotropic net-to-gross predictions for Woodside gas saturated reservoir 


\section{Discussion}

The results of this study demonstrate that ignoring anisotropy when making rock property predictions from seismic data can cause significant errors in both predicted porosity and predicted net-to-gross, in the order of 3\% and $40 \%$ respectively. As both porosity and net-to-gross are direct multipliers of reserve volume, this effect is economically significant.

Ignoring fracture anisotropy appears to have a greater effect on net-to-gross predictions than on porosity predictions. The ratio of maximum net-to-gross error to maximum porosity error was approximately 10:1. However, it is difficult to directly compare errors in porosity and net-to-gross. In reservoir terms, a 5\% predicted porosity error in a $15 \%$ porosity reservoir is proportionally equivalent to a $30 \%$ predicted net-to-gross error in a $100 \%$ net-to-gross reservoir.

It is important to note that the plots shown here are of the absolute error only, such as to quantify the maximum error value expected. Using the isotropic workflow in a fractured reservoir results in both under- and overestimations of rock properties. Both are of significance in making correct production decisions.

\section{Conclusions}

This study has demonstrated significant error resulting from rock property predictions using an isotropic workflow in a fractured reservoir, and showed that this effect warrants further investigation. The approach proposed in this paper presents an anisotropic alternative to the current standard isotropic rock property prediction workflow, incorporating the effects of shale anisotropy, layering anisotropy and fracturing anisotropy. This workflow can be used to make accurate rock property predictions from seismic data in an anisotropic reservoir situation.

\section{References}

Bakulin, A., Grechka, V., and Tsvankin, I., 2000, Estimation of fracture parameters from reflection seismic data - Part 1: HTI model due to a single fracture set: Geophysics, $\mathbf{6 5}$, 1788-1802.

Domenico, S. N., 1976, Effect of brine-gas mixture on velocity in an unconsolidated sand reservoir: Geophysics, 41, 882-894.

Gassmann, F., 1951, Über die elastizität poröser medien: Viertel. Naturforsch. Ges. Zürich, 96, 123.

Gurevich, B., 2002, Effect of the pore fluid on the elastic properties of a porous rock with parallel fractures: Journal of Expl. Geophys. (in print).

Mavko, G., Mukerji, T., and Dvorkin, J., 1998, The rock physics handbook (tools for seismic analysis in porous media): Cambridge University Press.

Rüger, A., 1998, Variation of P-wave reflectivity with offset and azimuth in anisotropic media: Geophysics, 63, 935-947.

Schoenberg, M., and Douma, J., 1988, Elastic-wave propagation in media with parallel fractures and aligned cracks: Geophys. Prosp., 36, 571-590

Schoenberg, M., and Muir, F., 1989, A calculus for finely layered anisotropic media: Geophysics, 54, 581-589.

Schoenberg, M., and Sayers, C. M., 1995, Seismic anisotropy of fractured rock: Geophysics, 60, 204-211.

Thomsen, L., 1986, Weak elastic anisotropy: Geophysics, 51, 1954-1966.

White, J. E., 1965, Seismic waves: radiation, transmission and attenuation: McGraw-Hill. 